\title{
CONVEXITÉ ET EQUATIONS DE CAUCHY-RIEMANN AVEC ESTIMATIONS $L^{p}$
}

\author{
Corinne Jouenne
}

\begin{abstract}
We obtain a solution of the equation $\bar{\partial} u=f$ as an integral supported only on the bounded convex domain $D$ of $\mathbb{C}^{n}$, without finite type assumption. We show that $u$ is in $L_{(0, q-1)}^{p}(D)$ if $f \in L_{(0, q)}^{p}(D)$ and $\frac{\bar{\partial} \rho \wedge f}{(-\rho)^{1-\varepsilon}} \in L_{(0, q+1)}^{p}(D)$ for some $\varepsilon$ depending on $p$ and $q, 0<\varepsilon \leq 1$.
\end{abstract}

\section{Introduction}

Considérons dans $\mathbb{C}^{n}, n \geq 2$, un domaine $D$ convexe, borné, de frontière $\partial D$ de classe $\mathcal{C}^{3}$ et de fonction définissante $\rho$, vérifiant $d \rho \neq 0$ sur $\partial D$. Nous nous intéressons à la résolution de l'équation de CauchyRiemann dans un tel domaine, sans hypothèse supplémentaire de type fini.

Parmi les résultats connus sur ce sujet, on peut citer les travaux de Chaumat et Chollet $[\mathbf{C . C}]$ qui construisent des noyaux intégraux explicites pour résoudre l'équation $\bar{\partial} u=f$ sur des convexes compacts $K$ de $\mathbb{C}^{n}, A$-normalisés (i.e. inclus dans la boule unité de $\mathbb{C}^{n}$ et contenant une boule fermée de rayon $A, A>0$ ). Lorsque la donnée $f$ est une $(0, q)$-forme $\bar{\partial}$-fermée et hölderienne sur $K$, ils obtiennent une solution $u$ vérifiant $\|u\|_{\mathcal{C}^{k, \alpha}(K)} \leq M\|f\|_{\mathcal{C}^{k+n-q, \alpha}(K)}$, où $M$ est une constante dépendant de $k, n, A$ et $\alpha$.

Dans $D \subset \mathbb{C}^{2}$, convexe à frontière $\mathcal{C}^{\infty}$, Range $[\mathbf{R} 1]$ construit un opérateur intégral $T: \mathcal{C}_{(0,1)}(\bar{D}) \rightarrow \mathcal{C}(D)$ solution pour $\bar{\partial}$, vérifiant

(i) $|T f|_{\Lambda_{\alpha}(D)} \leq C_{\alpha}|f|_{\Lambda_{\alpha}(D)}$ pour toute $f$ telle que $\bar{\partial} f=0$ et pour tout $\alpha>0$ et

(ii) $\|T f\|_{B M O(D)} \leq C\|f\|_{L^{\infty}}$.

1991 Mathematics Subject Classification. 32F20, 32F15, 32A25.

Key words. $\bar{\partial}$-equation, convex domain. 
D'autre part, Polking $[\mathbf{P}]$ résout dans les convexes bornés $D$ de $\mathbb{C}^{n}$ de fonction définissante $\rho$ de classe $\mathcal{C}^{\infty}$, l'équation $\bar{\partial} u=f$ avec l'estimation

$$
\left\||\rho|^{n-q-1-s} u\right\|_{L^{p}(D)} \leq C_{p}\left[\left\||\rho|^{1-s} f\right\|_{L^{p}(D)}+\left\||\rho|^{-s} \bar{\partial} \rho \wedge f\right\|_{L^{p}(D)}\right]
$$

pour $1 \leq q \leq n-1,1<p<+\infty$ et $0 \leq s \leq n-q-1$. En particulier, pour avoir une solution $u$ dans $L^{p}(D)$, il faut choisir $s=n-q-1$,

donc prendre une donnée $f$ telle que $\frac{f}{(-\rho)^{n-q-2}}$ et $\frac{f \wedge \bar{\partial} \rho}{(-\rho)^{n-q-1}}$ soient à coefficients dans $L^{p}(D)$. Ce résultat donne $L^{p} \rightarrow L^{p}$ pour $q=n-1$, mais la perte grandit avec $n-q$.

Nous nous sommes attachés à améliorer ce dernier résultat. Nous résolvons l'équation $\bar{\partial} u=f$ sous forme d'intégrale ne portant que sur le domaine $D$ défini au début de cette introduction. Pour une donnée satisfaisant une estimation $L^{p}$ mais avec la contrainte d'une meilleure régularité pour la composante tangentielle, nous obtenons une solution vérifiant une estimation $L^{p}, 1<p \leq \infty$.

\section{Notations et énoncé du théorème}

Rappelons une construction de l'opérateur solution de Henkin pour l'équation de Cauchy-Riemann, voir par exemple le livre de Range [R2]. Nous considérons un domaine $D=\left\{z \in \mathbb{C}^{n} / \rho(z)<0\right\}$, où la fonction définissante $\rho$ est convexe, $\mathcal{C}^{3}$ et vérifie $d \rho \neq 0$ sur $\partial D$.

Nous notons $\Phi(\zeta, z)=\langle\partial \rho(\zeta), \zeta-z\rangle$ la fonction support standard pour les domaines convexes. Comme $\Phi(\zeta, z) \neq 0$, pour $\zeta \in \partial D$ et $z \in D$, nous pouvons définir $S(\zeta, z)=\frac{\partial \rho(\zeta)}{\Phi(\zeta, z)}$.

L'application $s: \partial D \times D \rightarrow \mathbb{C}^{n}$, dont les composantes sont les coefficients de cette $\mathcal{C}_{(1,0)}^{1}$-forme, est une section du fibré de Cauchy-Leray au-dessus de $\partial D \times D$ qui vérifie $\langle s(\zeta, z), \zeta-z\rangle=1$.

Pour $\beta=\|\zeta-z\|^{2}, B=\frac{\partial \beta}{\beta}$ est la forme génératrice pour le noyau de Bochner-Martinelli-Koppelman, noté $K$.

Définissons aussi: $\widehat{S}(\zeta, \lambda, z)=\lambda S(\zeta, z)+(1-\lambda) B(\zeta, z)$ sur $\partial D \times$ $[0,1] \times D$, puis la forme de Cauchy-Fantappié: $\Omega(\widehat{S})=\frac{1}{(2 i \pi)^{n}} \widehat{S} \wedge\left(\bar{\partial}_{\zeta, z} \widehat{S}+\right.$ $\left.d_{\lambda} \widehat{S}\right)^{n-1}$.

Pour un noyau $L(\zeta, \lambda, z)$ ou $L(\zeta, z)$, nous noterons $L_{q}$ la composante de bidegré $(0, q)$ en $z$.

Rappelons que la solution de Henkin de l'équation $\bar{\partial} u=f$, pour $f$ une $(0, q)$-forme $\bar{\partial}$-fermée dans $D$, est donnée pour $1 \leq q \leq n-1$ par l'opérateur

$$
H_{q}: \mathcal{C}_{(0, q)}^{1}(\bar{D}) \longrightarrow \mathcal{C}_{(0, q-1)}^{1}(D)
$$

où $H_{q}$ est défini par $H_{q} f:=\int_{\partial D \times[0,1]} f \wedge \Omega_{q-1}(\widehat{S})-\int_{D} f \wedge K_{q-1}$. 
Dans la première intégrale, nous intégrons en $\lambda$ puis nous appliquons le théorème de Stokes après une modification du noyau que nous préciserons dans la démonstration du théorème. Ceci nous permet d'obtenir un opérateur $T_{q}$ ne faisant intervenir que des intégrales sur $D$.

Nous avons toutefois toujours:

$T_{q} f=H_{q} f$ et donc $\bar{\partial}\left(T_{q} f\right)=f$, pour toute $f \in \mathcal{C}_{(0, q)}^{1}(\bar{D}), f \bar{\partial}$-fermée.

Précisons quelques notations que nous emploierons au cours des calculs:

1. $\simeq$ signifiera que l'égalité est vraie "à un coefficient multiplicatif près".

$2 . \preceq$ sera utilisé pour indiquer que le terme de gauche d'une inégalité est majoré à une constante près par celui de droite.

Avec les notations précédentes, nous avons le

Théorème. Pour l'opérateur $T_{q}$, défini ci-dessus, $1 \leq q \leq n-1$, nous avons:

Si $f \in \mathcal{C}_{(0, q)}^{1}(\bar{D})$ et $\bar{\partial} f=0$, alors $\bar{\partial}\left(T_{q} f\right)=f$ sur $D$.

Pour $1 \leq q \leq n-2$, si de plus $f$ et $\frac{\bar{\partial} \rho \wedge f}{(-\rho)^{1-\varepsilon}}$ pour un $\varepsilon$ donné,

$$
0<\varepsilon \leq \frac{1-\frac{1}{p}-\theta}{n-q-\frac{1}{p}-\theta}
$$

où $\theta>0$ est arbitrairement petit, sont à coefficients dans $L^{p}(D)$, $1<p \leq+\infty$, alors $T_{q} f$ est à coefficients dans $L^{p}(D)$.

Pour $q=n-1$, nous avons $T_{q} f$ à coefficients dans $L^{p}(D)\left(\right.$ resp. $\left.L^{\infty}(D)\right)$ si $f$ et $\bar{\partial} \rho \wedge f$ (resp. $f$ et $\frac{\bar{\partial} \rho \wedge f}{(-\rho)^{1-\varepsilon}}, 0<\varepsilon<1$ ) sont à coefficients dans $L^{p}(D), 1<p<\infty$ (resp. $\left.L^{\infty}(D)\right)$.

Remarques. 1) L'opérateur $T_{q}$ ne dépend pas de $\varepsilon$.

2) Pour $q=n-1$ et $1<p<\infty$, le résultat vient du fait que l'on peut prendre $\varepsilon=1$ dans tous les calculs.

3) La méthode que nous utilisons est responsable du fait que la borne pour $\varepsilon$, donc le résultat, dépend de $p$, ce qui n'était pas le cas pour Chaumat et Chollet $[\mathbf{C . C}]$ et Polking $[\mathbf{P}]$. C'est aussi cette raison technique qui explique que le résultat est meilleur lorsque $p \rightarrow+\infty$ que lorsque $p \rightarrow 1$. Ceci était a priori surprenant puisque l'on assiste en général au phénomène contraire, comme dans les travaux de Fornæss et Sibony [F.S] qui obtiennent pour le $\bar{\partial}$, si $\Omega \subset \mathbb{C}$ et $1<p \leq 2$, des estimées $L^{p}$ à poids $e^{-\varphi}$, avec $\varphi$ sousharmonique; mais qui, pour $p>2$, construisent une fonction $\varphi$ 
sous-harmonique dans le disque unité $\Delta$ telle que, si $p^{\prime}>2$, l'image de l'opérateur $\bar{\partial}: L^{p^{\prime}}\left(\Delta, e^{-\varphi}\right) \rightarrow L^{p}\left(\Delta, e^{-\varphi}\right)$ n'est jamais fermée.

Ce théorème améliore pour $n-q \geq 2$ le résultat de Polking $[\mathbf{P}]$ rappelé dans l'introduction.

\section{Démonstration du théorème}

Nous commençons avec l'opérateur de Henkin rappelé dans le paragraphe 1:

$$
H_{q} f:=\int_{\partial D \times[0,1]} f \wedge \Omega_{q-1}(\widehat{S})-\int_{D} f \wedge K_{q-1}=J f+J^{0} f .
$$

$J^{0} f$ est une intégrale du type Bochner-Martinelli, donc clairement à coefficients dans $L^{p}(D)$ pour $f$ à coefficients dans $L^{p}(D)$.

Nous nous intéressons donc uniquement au terme $J f=\int_{\partial D \times[0,1]} f \wedge$ $\Omega_{q-1}(\widehat{S})$ et nous commençons par intégrer en $\lambda$.

En notant $I=[0,1]$, nous avons:

$$
\begin{aligned}
J f & =\int_{\partial D \times I} f \wedge \Omega_{q-1}(\widehat{S}) \simeq \int_{\partial D \times I} f \wedge \widehat{S} \wedge\left(\bar{\partial}_{\zeta} \widehat{S}+d_{\lambda} \widehat{S}\right)^{n-q} \wedge\left(\bar{\partial}_{z} \widehat{S}\right)^{q-1} \\
\simeq & \int_{\partial D \times I} f \wedge\left[\lambda \frac{\partial \rho}{\Phi}+(1-\lambda) B\right] \wedge\left\{\bar{\partial}_{\zeta}\left[\lambda \frac{\partial \rho}{\Phi}+(1-\lambda) B\right]\right\}^{n-q-1} \\
& \wedge\left[\frac{\partial \rho}{\Phi}-B\right] \wedge d \lambda \wedge\left\{\bar{\partial}_{z}[(1-\lambda) B]\right\}^{q-1} \operatorname{car} \bar{\partial}_{z}\left[\frac{\partial \rho(\zeta)}{\Phi(\zeta, z)}\right]=0 \\
\simeq & \int_{\partial D \times I} f \wedge B \wedge \frac{\partial \rho}{\Phi} \wedge\left\{\bar{\partial}_{\zeta}\left[\lambda \frac{\partial \rho}{\Phi}+(1-\lambda) B\right]\right\}^{n-q-1} \wedge d \lambda \wedge(1-\lambda)^{q-1}\left(\bar{\partial}_{z} B\right)^{q-1} \\
\simeq & \sum_{k=0}^{n-q-1} C_{k} \int_{\partial D \times I} f \wedge B \wedge \frac{\partial \rho}{\Phi} \wedge \lambda^{k}(1-\lambda)^{n-q-1-k}\left[\frac{\partial \partial \rho}{\Phi}-\frac{\partial \Phi \wedge \partial \rho}{\Phi^{2}}\right]^{k} \\
& \left.\simeq \sum_{k=0}^{n-q-1} C_{k}^{\prime} \int_{\partial D} f \wedge B \wedge \frac{\partial \rho}{\Phi} \wedge\left(\frac{\bar{\partial} \partial \rho}{\Phi}\right)^{k} B\right)^{n-q-1-k} \wedge d \lambda \wedge(1-\lambda)^{q-1}\left(\bar{\partial}_{z} B\right)^{q-1} \\
& \wedge\left(\bar{\partial}_{\zeta} B\right)^{n-q-1-k} \wedge\left(\bar{\partial}_{z} B\right)^{q-1} .
\end{aligned}
$$


Après cette intégration en $\lambda$, nous obtenons une somme de termes du type:

$$
\int_{\partial D} \frac{\left(\bar{\zeta}_{m_{1}}-\bar{z}_{m_{1}}\right)}{\|\zeta-z\|^{2(n-1-k)} \cdot \Phi(\zeta, z)^{k+1}} \frac{\partial \rho}{\partial \zeta_{m_{2}}}(\zeta) f(\zeta) \wedge \alpha_{M, k}(\zeta, z) \wedge \omega(\zeta),
$$

où $M=\left(m_{1}, \ldots, m_{n}\right), m_{i} \in\{1, \ldots, n\}, m_{i} \neq m_{j}$ si $i \neq j, \alpha_{M, k}(\zeta, z)$ est une forme de bidegré $(0, q-1)$ en $z$ et $(0, n-q-1)$ en $\zeta$, à coefficients $\mathcal{C}^{1}$, $0 \leq k \leq n-1, \omega(\zeta)=d \zeta_{1} \wedge \cdots \wedge d \zeta_{n}$.

Nous noterons pour $\zeta \in \bar{D}, z \in D$ :

$$
\begin{aligned}
\tilde{\Phi}_{\gamma, \varepsilon}(\zeta, z) & :=\Phi(\zeta, z)-2 \rho(\zeta)+[-\rho(\zeta)+\gamma]^{\varepsilon}\|\zeta-z\|^{2} \text {, où } \gamma>0 \\
\tilde{\Phi}_{\varepsilon}(\zeta, z) & :=\lim _{\gamma \rightarrow 0} \tilde{\Phi}_{\gamma, \varepsilon}(\zeta, z) \quad \text { et } \quad \tau(\zeta, z):=\|\zeta-z\|^{2}+\rho(\zeta) \rho(z) .
\end{aligned}
$$

Remarquons que, pour $\zeta \in \partial D$, nous avons:

$$
\tilde{\Phi}_{\varepsilon}(\zeta, z)=\Phi(\zeta, z) \quad \text { et } \quad \tau(\zeta, z)=\|\zeta-z\|^{2} .
$$

Dans la suite, nous aurons besoin des minorations suivantes:

$$
\left|\operatorname{Re}\left(\tilde{\Phi}_{\varepsilon}(\zeta, z)\right)\right| \geq C_{1}\left(-\rho(\zeta)-\rho(z)+(-\rho(\zeta))^{\varepsilon}\|\zeta-z\|^{2}\right)
$$

et

$$
\tau(\zeta, z) \geq C_{2}\left(\|\zeta-z\|^{2}+\rho(\zeta)^{2}+\rho(z)^{2}\right)
$$

où $C_{1}$ et $C_{2}$ sont deux constantes positives. La première de ces minorations vient de la définition de $\Phi$ et du fait que $\rho$ est convexe, la seconde est immédiate, puisque $|\rho(\zeta)-\rho(z)| \preceq\|\zeta-z\|$.

Nous notons $J_{\gamma, \varepsilon} f$ (resp. $J_{\varepsilon} f$ ) l'intégrale obtenue à partir de $J f$ en remplaçant $\|\zeta-z\|^{2}$ par $\tau(\zeta, z)$, dans le dénominateur de $B$, et $\Phi$ par $\tilde{\Phi}_{\gamma, \varepsilon}\left(\operatorname{resp} . \Phi \operatorname{par} \tilde{\Phi}_{\varepsilon}\right)$.

Après l'intégration en $\lambda$, nous appliquons le théorème de Stokes à chacune des intégrales qui composent $J_{\gamma, \varepsilon} f$, en remarquant qu'il n'intervient que des dérivations en $\bar{\partial}$, puisque la forme différentielle à intégrer est complète en $d \zeta$, et en tenant compte du fait que $f$ est $\bar{\partial}$-fermée. Nous faisons alors tendre $\gamma$ vers 0 . $\left(J_{\gamma, \varepsilon} f\right.$ tend vers $J_{\varepsilon} f$ lorsque $\gamma$ tend vers 0 par un argument de convergence dominée, en utilisant les minorations données ci-dessus.) 
Nous devons maintenant estimer des termes des types suivants:

$$
\begin{aligned}
& A_{1}(k)=\int_{D} \frac{|f(\zeta)|}{\left|\tilde{\Phi}_{\varepsilon}\right|^{k+1} \tau(\zeta, z)^{n-1-k}} d \lambda(\zeta) \\
& A_{2}(k)=\int_{D} \frac{|f(\zeta)|\|\zeta-z\|^{2}}{\left|\tilde{\Phi}_{\varepsilon}\right|^{k+2} \tau(\zeta, z)^{n-1-k}} d \lambda(\zeta) \\
& A_{3}(k)=\int_{D} \frac{|f(\zeta) \wedge \bar{\partial} \rho(\zeta)|\|\zeta-z\|^{3}}{(-\rho(\zeta))^{1-\varepsilon}\left|\tilde{\Phi}_{\varepsilon}\right|^{k+2} \tau(\zeta, z)^{n-1-k}} d \lambda(\zeta) \\
& A_{4}(k)=\int_{D} \frac{|f(\zeta) \wedge \bar{\partial} \rho(\zeta)|\|\zeta-z\|}{\left|\tilde{\Phi}_{\varepsilon}\right|^{k+2} \tau(\zeta, z)^{n-1-k}} d \lambda(\zeta)
\end{aligned}
$$

où $0 \leq k \leq n-q-1$, les autres termes obtenus étant plus réguliers. Pour simplifier les notations, nous omettrons le $\varepsilon$ en indice dans la suite du texte.

Rappelons qu'il suffit de s'assurer des bonnes estimations pour les cas estrêmes $k=0$ et $k=n-q-1$, d'après un résultat d'analyse réelle démontré dans un article de Krantz [K, Théorème 4.6, p. 239] de 1976.

- Nous utilisons pour cela, lorsque $1<p<+\infty$, comme Polking [P], un lemme de type Shur. Rappelons la version de Foreli-Rudin $[\mathbf{F . R}]$ :

Lemme 1 ([F.R]). Soit $\mu$ une mesure positive sur un espace mesurable $X$.

Soient $Q: X \times X \rightarrow[0,+\infty)$ mesurable, $1<p<+\infty, \frac{1}{p}+\frac{1}{p^{\prime}}=1$. Supposons qu'il existe une fonction mesurable $g: X \rightarrow(0,+\infty)$ et des constantes $a$ et $b$ pour lesquelles on a les estimations:

$$
\int_{X} Q(x, y) g(y)^{p^{\prime}} d \mu(y) \leq[a g(x)]^{p^{\prime}} \quad(x \in X)
$$

et

$$
\int_{X} Q(x, y) g(x)^{p} d \mu(x) \leq[b g(y)]^{p} \quad(y \in X) .
$$

Alors l'équation $(T f)(x)=\int_{X} Q(x, y) f(y) d \mu(y)$ définit un opérateur borné $T$ sur $L^{p}(\mu)$, avec $\|T\| \leq a b$.

Remarque. Ceci n'est pas la version la plus générale du Lemme de Shur, mais une étude calculatoire permet de voir que c'est dans le cas particulier que nous avons rappelé que l'on obtient les meilleures bornes pour $\varepsilon$. 
Nous partageons dans toutes les intégrales le domaine $D$ en un domaine

$$
\tilde{D}_{z}=\left\{\zeta \in D,|\tilde{\Phi}(\zeta, z)|+\|\zeta-z\|<\theta_{0}\right\}, \text { où } 0<\theta_{0} \ll 1
$$

et un domaine sur lequel le noyau est intégrable en $\zeta$, et ce uniformément en $z$. Nous appliquons le lemme pour le terme $A_{1}(0)$ avec $Q(\zeta, z)=$ $\frac{1}{\tau(\zeta, z)^{n-1} \cdot|\tilde{\Phi}(\zeta, z)|}$ et $g \equiv 1$ sur $D$.

Dans toutes les autres intégrales, nous employons le Lemme 1 avec la fonction $g$ définie sur $D$ par $g(\zeta)=\frac{1}{(-\rho(\zeta))^{\frac{1}{p p^{\prime}}}}$.

Nous étudions uniquement l'intégrale sur $\tilde{D}$ et introduisons pour plus de clarité

$$
I_{(a, b, c, d)}=\int_{\tilde{D}} \frac{g(\zeta)^{p^{\prime}}\|\zeta-z\|^{a}(-\rho(\zeta))^{b}}{\tau(\zeta, z)^{c} \cdot|\tilde{\Phi}(\zeta, z)|^{d}} d \lambda(\zeta)
$$

et

$$
L_{(a, b, c, d)}=\int_{\tilde{D}} \frac{g(z)^{p}\|\zeta-z\|^{a}(-\rho(\zeta))^{b}}{\tau(\zeta, z)^{c} \cdot|\tilde{\Phi}(\zeta, z)|^{d}} d \lambda(z) .
$$

Nous appliquons alors le Lemme 1 avec $Q(\zeta, z)=\frac{1}{\tau(\zeta, z)^{q} \cdot|\tilde{\Phi}(\zeta, z)|^{n-q}}$ pour le terme $A_{1}(n-q-1)$, ce qui revient à étudier les intégrales $I_{(0,0, q, n-q)}$ et $L_{(0,0, q, n-q)}$ que l'on trouvera dans le Lemme 4 , pour $1 \leq q \leq n-2$. Le cas $q=n-1$ peut se traiter en prenant $g \equiv 1$ sur $D$ et on obtient alors les mêmes intégrales que pour $A_{1}(0)$.

Pour l'intégrale $A_{2}(0)$, nous avons $I_{(2,0, n-1,2)}$ et $L_{(2,0, n-1,2)}$, intégrales respectivement contrôlées par $I_{(1,0, n-1,2)}$ et $L_{(1,0, n-1,2)}$ qui sont estimées dans le Lemme 5 , et pour $A_{2}(n-q-1)$, les intégrales obtenues $I_{(2,0, q, n-q+1)}$ et $L_{(2,0, q, n-q+1)}$ sont majorées dans le Lemme 3 .

Sous l'hypothèse que $\frac{f \wedge \bar{\partial} \rho}{(-\rho)^{1-\varepsilon}}$ est à coefficients dans $L^{p}(D)$, les termes $A_{3}(0)$ et $A_{3}(n-q-1)$ sont plus réguliers que ceux obtenus pour $A_{2}(0)$ et $A_{2}(n-q-1)$.

Enfin, pour $A_{4}(0)$, nous étudions les intégrales $I_{(1,0, n-1,2)}$ et $L_{(1,0, n-1,2)}$ dans le Lemme 5 (après avoir remarqué que $I_{(1,1-\varepsilon, n-1,2)} \preceq$ $I_{(1,0, n-1,2)}$ et $\left.L_{(1,1-\varepsilon, n-1,2)} \preceq L_{(1,0, n-1,2)}\right)$. Le terme $A_{4}(n-q-1)$ se traite de façon similaire.

Il apparait au cours des calculs que, pour $1<p<\infty$, $\varepsilon$ doit être choisi tel que

$$
0<\varepsilon \leq \frac{1-\frac{1}{p}-\theta}{n-q-\frac{1}{p}-\theta},
$$

où $\theta>0$ est arbitrairement petit. 
Remarquons que $\varepsilon$ dépend de $p$ et $q$.

Pour toutes les estimations, le résultat découle du Lemme 1 , avec le changement de variables $\left(t_{1}, t_{2}, \ldots, t_{2 n}\right)$, où nous noterons $t^{\prime}=\left(t_{3}, \ldots, t_{2 n}\right)$ et avec $t_{1}=-\rho(\zeta)($ resp. $-\rho(z))$ et $t_{2}=\operatorname{Im} \Phi$ dans les intégrales en $\zeta$ (resp. en $z$ ).

Ce changement de variables est valide grâce à la convexité de $D$, comme le montre le Lemme 2 dû à Polking $[\mathbf{P}]$.

Lemme $2([\mathbf{P}])$. La fonction $\Phi: \bar{D} \times \bar{D} \rightarrow \mathbb{C}$ a les propriétés suivantes:

1) $\Phi \in \mathcal{C}^{\infty}(\bar{D} \times \bar{D})$ et $\Phi(\zeta,.) \in \mathcal{O}(D)$ pour tout $\zeta \in \bar{D}$.

2) $2 \operatorname{Re} \Phi(\zeta, z) \geq-\rho(\zeta)-\rho(z)$ pour tous $\zeta, z \in \bar{D}$.

3) Supposons que $\Phi\left(\zeta_{0}, z_{0}\right)=0,\left(\zeta_{0}, z_{0}\right) \in \bar{D} \times \bar{D}$. Alors $\zeta_{0}, z_{0} \in \partial D$ et il existe des champs de vecteurs $X_{\zeta}$ en $\zeta$ et $Y_{z}$ en $z$ tangents $\grave{a} \partial D$ en $\zeta_{0}$ et $z_{0}$ respectivement tels que $\left(X_{\zeta} \operatorname{Im} \Phi\right)\left(\zeta_{0}, z_{0}\right) \neq 0$ et $\left(Y_{z} \operatorname{Im} \Phi\right)\left(\zeta_{0}, z_{0}\right) \neq 0$.

La propriété 3) de ce lemme vient du fait que $\Phi$ est une fonction support pour $D$ et utilise la convexité du domaine. Ceci nous permet de prendre $\rho$ et $\operatorname{Im} \Phi$ comme variables réelles indépendantes près des points où $\Phi$ s'annule.

- Traitons maintenant le cas $p=+\infty$.

Nous adapterons la notation

$$
K_{(a, b, c, d)}=K_{(a, b, c, d)}(z)=\int_{\tilde{D}} \frac{\|\zeta-z\|^{a}(-\rho(\zeta))^{b}}{\tau(\zeta, z)^{c} \cdot|\tilde{\Phi}(\zeta, z)|^{d}} d \lambda(\zeta), \text { où } \tilde{D}=\tilde{D}_{z} .
$$

Considérons d'abord les termes $A_{1}$ et $A_{2}$, avec la condition que $f$ est à coefficients dans $L^{\infty}(D)$.

Pour $A_{1}(0)$

$$
\forall z \in D, \int_{\tilde{D}} \frac{|f(\zeta)| d \lambda(\zeta)}{\tau(\zeta, z)^{n-1} \cdot|\tilde{\Phi}(\zeta, z)|} \preceq\|f\|_{\infty} \cdot K_{(0,0, n-1,1)} \preceq\|f\|_{\infty} .
$$

De même, nous avons pour $A_{1}(n-q-1)$ :

$$
\forall z \in D, \int_{\tilde{D}} \frac{|f(\zeta)| d \lambda(\zeta)}{\tau(\zeta, z)^{q} \cdot|\tilde{\Phi}(\zeta, z)|^{n-q}} \preceq\|f\|_{\infty} \cdot K_{(0,0, q, n-q)} \preceq\|f\|_{\infty},
$$

d'après le Lemme 7 pour $1 \leq q \leq n-2$ et de façon claire pour $q=n-1$.

Pour $A_{2}(0)$

$$
\int_{\tilde{D}} \frac{|f(\zeta)|\|\zeta-z\|^{2} d \lambda(\zeta)}{\tau(\zeta, z)^{n-1} \cdot|\tilde{\Phi}(\zeta, z)|^{2}} \preceq\|f\|_{\infty} \cdot K_{(2,0, n-1,2)} .
$$


Et pour $A_{2}(n-q-1)$

$$
\int_{\tilde{D}} \frac{|f(\zeta)|\|\zeta-z\|^{2} d \lambda(\zeta)}{\tau(\zeta, z)^{q} \cdot|\tilde{\Phi}(\zeta, z)|^{n-q+1}} \preceq\|f\|_{\infty} \cdot K_{(2,0, q, n-q+1)} .
$$

Les deux intégrales des membres de droite sont majorées dans le Lemme 6 , en choisissant $0<\varepsilon<\frac{1}{n-q}$.

Remarque. En particulier pour $q=n-1$, ceci nous donne $0<\varepsilon<1$ et c'est la raison pour laquelle, même dans ce cas, nous n'obtenons pas la résolution de l'équation $\bar{\partial} u=f$ avec une solution dans $L^{\infty}(D)$, pour une donnée dans $L^{\infty}(D)$.

Avec la condition que $\frac{f \wedge \bar{\partial} \rho}{(-\rho)^{1-\varepsilon}}$ est à coefficients dans $L^{\infty}(D)$, on étudierait de même les termes $A_{3}$ et $A_{4}$.

\section{Lemmes techniques}

Nous donnons dans ce paragraphe les détails techniques qui justifient les résultats précédents.

Nous noterons: $\eta=\frac{1}{p}, \gamma=\frac{1}{p^{\prime}}$, avec $\frac{1}{p}+\frac{1}{p^{\prime}}=1$.

Donnons maintenant les estimations des intégrales qui apparaissent dans la démonstration pour le cas $L^{p}, 1<p<\infty$. Nous énonçons tout d'abord celles pour lesquelles nous obtenons les bornes de $\varepsilon$ données dans le théorème et qui proviennent du terme $A_{2}(n-q-1)$.

Lemme 3. Pour $1 \leq q \leq n-1$, il existe $C_{1}$ et $C_{2}$ des constantes positives telles que

$$
I_{(2,0, q, n-q+1)} \leq \frac{C_{1}}{(-\rho(z))^{\eta}} \quad \text { pour tout } z \in D
$$

et

$$
L_{(2,0, q, n-q+1)} \leq \frac{C_{2}}{(-\rho(\zeta))^{\gamma}} \quad \text { pour tout } \zeta \in D,
$$

pour

$$
0<\varepsilon<\frac{1-\eta}{n-q-\eta} \quad \text { si } 1 \leq q \leq n-2
$$

et pour

$$
0<\varepsilon \leq 1 \quad \text { si } q=n-1 .
$$

Démonstration: Le cas $q=n-1$ est une conséquence du Lemme 5. Nous avons ainsi les bonnes estimations pour tout $\varepsilon, 0<\varepsilon \leq 1$.

Il nous reste donc à considérer le cas $1 \leq q \leq n-2$ : 
(a) Nous avons, avec le changement de variables $\left(t_{1}, t_{2}, \ldots, t_{2 n}\right)$, où $t_{1}=-\rho(\zeta), t_{2}=\operatorname{Im} \Phi, t^{\prime}=\left(t_{3}, \ldots, t_{2 n}\right)$, en notant $a=-\rho(z)$ et en intégrant en $t_{2}$ puis en coordonnées polaires sur $t^{\prime}$ :

$$
\begin{aligned}
& I_{(2,0, q, n-q+1)} \\
& =\int_{\tilde{D}} \frac{\|\zeta-z\|^{2} d \lambda(\zeta)}{(-\rho(\zeta))^{\eta}\left[\|\zeta-z\|^{2}+\rho(\zeta) \rho(z)\right]^{q}\left[-\rho(\zeta)-\rho(z)+|\operatorname{Im} \Phi|+(-\rho(\zeta))^{\varepsilon}\|\zeta-z\|^{2}\right]^{n-q+1}} \\
& \preceq \int_{0<t_{1}<1} \int_{\left|t_{2}\right|<1} \int_{\left|t^{\prime}\right|<1} \frac{\left|t^{\prime}\right|^{2 n-3} d t_{1} d t_{2} d\left|t^{\prime}\right|}{t_{1}^{\eta}\left|t^{\prime}\right|^{2 q-2}\left[t_{1}+a+\left|t_{2}\right|+t_{1}^{\varepsilon}\left|t^{\prime}\right|^{2}\right]^{n-q+1}} \\
& \preceq \int_{0<t_{1}<1} \int_{\left|t^{\prime}\right|<1} \frac{\left|t^{\prime}\right|^{2 n-2 q-1} d t_{1} d\left|t^{\prime}\right|}{t_{1}^{\eta}\left[t_{1}+a+t_{1}^{\varepsilon}\left|t^{\prime}\right|^{2}\right]^{n-q}} \\
& \preceq \frac{1}{a^{\eta}} \int_{0<t_{1}<1} \int_{\left|t^{\prime}\right|<1} \frac{\left|t^{\prime}\right|^{2 n-2 q-1} d t_{1} d\left|t^{\prime}\right|}{t_{1}^{\eta} t_{1}^{\varepsilon(n-q-\eta)}\left|t^{\prime}\right|^{2 n-2 q-2 \eta}} \\
& \preceq \frac{1}{a^{\eta}} \int_{0<t_{1}<1} \int_{\left|t^{\prime}\right|<1} \frac{d t_{1} d\left|t^{\prime}\right|}{t_{1}^{\eta+\varepsilon(n-q-\eta)}\left|t^{\prime}\right|^{1-2 \eta}} \\
& \preceq \frac{1}{(-\rho(z))^{\frac{1}{p}}}
\end{aligned}
$$

pour $0<\varepsilon<\frac{1-\frac{1}{p}}{n-q-\frac{1}{p}}$.

(b) Nous obtenons de même, en posant $t_{1}=-\rho(z)$ et $t_{2}=\operatorname{Im} \Phi$ et en notant $a=-\rho(\zeta)$ :

$$
\begin{aligned}
& L_{(2,0, q, n-q+1)} \\
& =\int_{\tilde{D}} \frac{\|\zeta-z\|^{2} d \lambda(z)}{(-\rho(z))^{\gamma}\left[\|\zeta-z\|^{2}+\rho(\zeta) \rho(z)\right]^{q}\left[-\rho(\zeta)-\rho(z)+|\operatorname{Im} \Phi|+(-\rho(\zeta))^{\varepsilon}\|\zeta-z\|^{2}\right]^{n-q+1}} \\
& \preceq \int_{0<t_{1}<1} \int_{\left|t^{\prime}\right|<1} \frac{\left|t^{\prime}\right|^{2 n-2 q-1} d t_{1} d\left|t^{\prime}\right|}{t_{1}^{\gamma}\left[a+t_{1}+a^{\varepsilon}\left|t^{\prime}\right|^{2}\right]^{n-q}} \\
& \preceq \int_{0<t_{1}<1} \int_{\left|t^{\prime}\right|<1} \frac{\left|t^{\prime}\right|^{2 n-2 q-1} d t_{1} d\left|t^{\prime}\right|}{t_{1}^{\gamma+\alpha} a^{\varepsilon(n-q-\alpha)}\left|t^{\prime}\right|^{2 n-2 q-2 \alpha}} \quad \text { où } 0 \leq \alpha \leq n-q \\
& \preceq \frac{1}{a^{\varepsilon(n-q-\alpha)}} \int_{0<t_{1}<1} \int_{\left|t^{\prime}\right|<1} \frac{d t_{1} d\left|t^{\prime}\right|}{t_{1}^{\gamma+\alpha}\left|t^{\prime}\right|^{1-2 \alpha}} \\
& \preceq \frac{1}{(-\rho(\zeta))^{\frac{1}{p^{\prime}}}}
\end{aligned}
$$

pour $0<\alpha<1-\gamma=\frac{1}{p}$ et pour $0<\varepsilon \leq \frac{1-\frac{1}{p}}{n-q-\alpha}$, soit finalement pour $0<\varepsilon<\frac{1-\frac{1}{p}}{n-q-\frac{1}{p}}$ 
Ensuite nous étudions les intégrales qui interviennent dans les termes $A_{1}(n-q-1)$ et $A_{4}(n-q-1)$ et pour lesquelles nous avons également des conditions sur $\varepsilon$.

Lemme 4. Pour $1 \leq q \leq n-2(n \geq 3)$, il existe $C_{1}$ et $C_{2}$ des constantes positives telles que, pour tout $\varepsilon, 0<\varepsilon<\frac{1-\eta}{n-q-1-\eta}$ :

$$
I_{(0,0, q, n-q)} \leq \frac{C_{1}}{(-\rho(z))^{\eta}}
$$

et

$$
L_{(0,0, q, n-q)} \leq \frac{C_{2}}{(-\rho(\zeta))^{\gamma}}
$$

Démonstration: $(a)$ En posant $t_{1}=-\rho(\zeta)$ et $t_{2}=\operatorname{Im} \Phi$ et en notant $a=-\rho(z)$, nous avons:

$$
=\int_{\tilde{D}} \frac{d \lambda(\zeta)}{(-\rho(\zeta))^{\eta}\left[\|\zeta-z\|^{2}+\rho(\zeta) \rho(z)\right]^{q}\left[-\rho(\zeta)-\rho(z)+|\operatorname{Im} \Phi|+(-\rho(\zeta))^{\varepsilon}\|\zeta-z\|^{2}\right]^{n-q}} .
$$

Donc

$$
\begin{aligned}
I_{(0,0, q, n-q)} & \int_{0<t_{1}<1} \int_{\left|t_{2}\right|<1} \int_{\left|t^{\prime}\right|<1} \frac{\left|t^{\prime}\right|^{2 n-2 q-3} d t_{1} d t_{2} d\left|t^{\prime}\right|}{t_{1}^{\eta}\left[t_{1}-\rho(z)+\left|t_{2}\right|+t_{1}^{\varepsilon}\left|t^{\prime}\right|^{2}\right]^{n-q}} \\
& \preceq \int_{0<t_{1}<1} \int_{\left|t^{\prime}\right|<1} \frac{\left|t^{\prime}\right|^{2 n-2 q-3} d t_{1} d\left|t^{\prime}\right|}{t_{1}^{\eta}\left[t_{1}+a+t_{1}^{\varepsilon}\left|t^{\prime}\right|^{2}\right]^{n-q-1}} \\
& \preceq \frac{1}{a^{\eta}} \int_{0<t_{1}<1} \int_{\left|t^{\prime}\right|<1} \frac{\left|t^{\prime}\right|^{2 n-2 q-3} d t_{1} d\left|t^{\prime}\right|}{t_{1}^{\eta} t_{1}^{\varepsilon(n-q-1-\eta)}\left|t^{\prime}\right|^{2 n-2 q-2-2 \eta}} \\
& \preceq \frac{1}{a^{\eta}} \int_{0<t_{1}<1} \int_{\left|t^{\prime}\right|<1} \frac{d t_{1} d\left|t^{\prime}\right|}{t_{1}^{\eta+\varepsilon(n-q-1-\eta)}\left|t^{\prime}\right|^{1-2 \eta}} \\
& \preceq \frac{1}{(-\rho(z))^{\frac{1}{p}}} \text { pour } 0<\varepsilon<\frac{1-\eta}{n-q-1-\eta} .
\end{aligned}
$$


(b) Nous obtenons de même, en posant $t_{1}=-\rho(z)$ et $t_{2}=\operatorname{Im} \Phi$ :

$$
\begin{aligned}
& L_{(0,0, q, n-q)} \\
& =\int_{\tilde{D}} \frac{d \lambda(z)}{(-\rho(z))^{\gamma}\left[\|\zeta-z\|^{2}+\rho(\zeta) \rho(z)\right]^{q}\left[-\rho(\zeta)-\rho(z)+|\operatorname{Im} \Phi|+(-\rho(\zeta))^{\varepsilon}\|\zeta-z\|^{2}\right]^{n-q}} \\
& \preceq \int_{0<t_{1}<1} \int_{\left|t^{\prime}\right|<1} \frac{\left|t^{\prime}\right|^{2 n-2 q-3} d t_{1} d\left|t^{\prime}\right|}{t_{1}^{\gamma}\left[t_{1}-\rho(\zeta)+(-\rho(\zeta))^{\varepsilon}\left|t^{\prime}\right|^{2}\right]^{n-q-1}} \\
& \preceq \frac{1}{a^{\gamma}} \int_{0<u_{1}<1} \int_{\left|t^{\prime}\right|<1} \frac{a^{1-\eta}\left|t^{\prime}\right|^{2 n-2 q-3} d u_{1} d\left|t^{\prime}\right|}{u_{1}^{\gamma}\left[a+a^{\varepsilon}\left|t^{\prime}\right|^{2}\right]^{n-q-1-\eta}} \\
& \quad+\frac{1}{a^{\gamma}} \int_{1 \leq u_{1}<\frac{1}{a}} \int_{\left|t^{\prime}\right|<1} \frac{a^{1-\alpha}\left|t^{\prime}\right|^{2 n-2 q-3} d u_{1} d\left|t^{\prime}\right|}{u_{1}^{\gamma+\alpha}\left[a+a^{\varepsilon}\left|t^{\prime}\right|^{2}\right]^{n-q-1-\alpha}}
\end{aligned}
$$

avec $a=-\rho(\zeta), t_{1}=a u_{1}$ et $1-\gamma<\alpha \leq 1$

$$
\begin{aligned}
& \preceq \frac{1}{a^{\gamma}} \int_{0<u_{1}<1} \int_{\left|t^{\prime}\right|<1} \frac{a^{1-\eta-\varepsilon(n-q-1-\eta)} d u_{1} d\left|t^{\prime}\right|}{u_{1}^{\gamma}\left|t^{\prime}\right|^{1-2 \eta}} \\
& \quad+\frac{1}{a^{\gamma}} \int_{1 \leq u_{1}<\frac{1}{a}} \int_{\left|t^{\prime}\right|<1} \frac{a^{1-\alpha-\varepsilon(n-q-1-\alpha)} d u_{1} d\left|t^{\prime}\right|}{u_{1}^{\gamma+\alpha}\left|t^{\prime}\right|^{1-2 \alpha}} \\
& \preceq \frac{1}{(-\rho(\zeta))^{1} p^{\prime}}
\end{aligned}
$$

pour $\alpha=1-\gamma+\theta=\frac{1}{p}+\theta$ et $0<\varepsilon \leq \frac{1-\frac{1}{p}-\theta}{n-q-1-\frac{1}{p}-\theta}$ avec $\theta>0$ arbitrairememnt petit, sout pour tout $\varepsilon, 0<\varepsilon<\frac{1-\frac{1}{p}}{n-q-1-\frac{1}{p}}$.

Enfin, nous achevons le cas $1<p<\infty$ avec les intégrales plus simples données par l'étude des termes $A_{4}(0)$ et $A_{1}(0)$ et qui autorisent à prendre $0<\varepsilon \leq 1$.

Lemme 5. Il existe $C_{1}$ et $C_{2}$ des constantes positives telles que pour tout $\varepsilon, 0<\varepsilon \leq 1$

$$
I_{(1,0, n-1,2)} \leq \frac{C_{1}}{(-\rho(z))^{\eta}}
$$

et

$$
L_{(1,0, n-1,2)} \leq \frac{C_{2}}{(-\rho(\zeta))^{\gamma}}
$$


Démonstration: (a) En posant $t_{1}=-\rho(\zeta)$ et $t_{2}=\operatorname{Im} \Phi$, et en notant $a=-\rho(z)$ :

$$
\begin{aligned}
& I_{(1,0, n-1,2)} \\
& =\int_{\tilde{D}} \frac{\|\zeta-<\| d \lambda(\zeta)}{(-\rho(\zeta))^{\eta}\left[\|\zeta-z\|^{2}+\rho(\zeta) \rho(z)\right]^{n-1}\left[-\rho(\zeta)-\rho(z)+|\operatorname{Im} \Phi|+(-\rho(\zeta))^{\varepsilon}\|\zeta-z\|^{2}\right]^{2}} \\
& \preceq \int_{0<t_{1}<1} \int_{\left|t_{2}\right|<1} \int_{\left|t^{\prime}\right|<1} \frac{\left|t^{\prime}\right|^{2 n-3} d t_{1} d t_{2} d\left|t^{\prime}\right|}{t_{1}^{\eta}\left|t^{\prime}\right|^{2 n-3}\left[t_{1}+a+\left|t_{2}\right|+t_{1}^{\varepsilon}\left|t^{\prime}\right|^{2}\right]^{2}} \\
& \preceq \int_{0}^{1} \frac{d t_{1}}{t_{1}^{\eta}\left(t_{1}+a\right)} \\
& \preceq \frac{1}{(-\rho(z))^{\frac{1}{p}}}
\end{aligned}
$$

après intégration par parties, comme dans le Lemme 1.6.

(b) De même, en posant $t_{1}=-\rho(z), t_{2}=\operatorname{Im} \Phi$ et $a=-\rho(\zeta)$, nous avons:

$$
\begin{aligned}
& L_{(1,0, n-1,2)} \\
& =\int_{\tilde{D}} \frac{\|\zeta-z\| d \lambda(z)}{(-\rho(z))^{\gamma}\left[\|\zeta-z\|^{2}+\rho(\zeta) \rho(z)\right]^{n-1}\left[-\rho(\zeta)-\rho(z)+|\operatorname{Im} \Phi|+(-\rho(\zeta))^{\varepsilon}\|\zeta-z\|^{2}\right]^{2}} \\
& \preceq \int_{0<t_{1}<1} \int_{\left|t_{2}\right|<1} \int_{\left|t^{\prime}\right|<1} \frac{\left|t^{\prime}\right|^{2 n-3} d t_{1} d t_{2} d\left|t^{\prime}\right|}{t_{1}^{\gamma}\left|t^{\prime}\right|^{2 n-3}\left[t_{1}+a+a^{\varepsilon}\left|t^{\prime}\right|^{2}\right]^{2}} \\
& \preceq \int_{0<t_{1}<1} \int_{\left|t^{\prime}\right|<1} \frac{d t_{1} d\left|t^{\prime}\right|}{t_{1}^{\gamma}\left(t_{1}+a\right)} \\
& \preceq \frac{1}{(-\rho(\zeta))^{\frac{1}{p^{\prime}}}} \\
& \text { comme précédemment. }
\end{aligned}
$$

Pour achever ce paragraphe, nous donnons les majorations des intégrales à considérer dans le cas $p=+\infty$. Remarquons que les termes $A_{j}(0), 1 \leq j \leq 4$, seront toujours obtenus comme cas particuliers de $A_{j}(n-q-1)$ pour $q=n-1$ et ne seront donc pas estimés séparément.

Nous commençons à nouveau par les intégrales qui font apparaître les bornes imposées à $\varepsilon$ dans l'énoncé du théorème et qui proviennent de $A_{2}(n-q-1)$.

Lemme 6. Pour $1 \leq q \leq n-1$, il existe $C$ une constante positive telle que

pour tout $\varepsilon, 0<\varepsilon<\frac{1}{n-q}$.

$$
K(2,0, q, n-q+1) \leq C
$$


Démonstration: En posant $t_{1}=-\rho(\zeta)$ et $t_{2}=\operatorname{Im} \Phi$, nous avons:

$$
K_{(2,0, q, n-q+1)}=\int_{\tilde{D}} \frac{\mid f(\zeta)\|\zeta-z\|^{2} d \lambda(\zeta)}{\tau(\zeta, z)^{q} \cdot|\tilde{\Phi}(\zeta, z)|^{n-q+1}} \preceq\|f\|_{\infty} I_{2}
$$

où

$$
\begin{aligned}
I_{2} & \preceq \int_{0<t_{1}<1} \int_{\left|t^{\prime}\right|<1} \frac{\left|t^{\prime}\right|^{2 n-2 q-1} d t_{1} d\left|t^{\prime}\right|}{\left[t_{1}-\rho(z)+t_{1}^{\varepsilon}\left|t^{\prime}\right|^{2}\right]^{n-q}} \\
& \preceq \int_{0<t_{1}<1} \int_{\left|t^{\prime}\right|<1} \frac{d t_{1} d\left|t^{\prime}\right|}{\theta+\varepsilon(n-q-\theta)\left|t^{\prime}\right|^{1-2 \theta}} \\
& \leq C
\end{aligned}
$$

pour $0<\varepsilon<\frac{1-\theta}{n-q-\theta}$, avec $\theta>0$ arbitrairement petit, soit pour tout $\varepsilon$, $0<\varepsilon<\frac{1}{n-q}$.

Enfin, nous avons l'intégrale donnée par le terme $A_{1}(n-q-1)$ qui permet de prendre $0<\varepsilon<\frac{1}{n-q-1}$ pour $1 \leq q \leq n-2$.

Lemme 7. Pour $1 \leq q \leq n-2$, il existe $C$ une constante positive telle que

$$
K_{(0,0, q, n-q)} \leq C
$$

pour tout $\varepsilon, 0<\varepsilon<\frac{1}{n-q-1}$.

Démonstration: Avec le changement de variables $t_{1}=-\rho(\zeta)$ et $t_{2}=$ $\operatorname{Im} \Phi$, nous avons:

$$
K_{(0,0, q, n-q)}=\int_{\tilde{D}} \frac{|f(\zeta)| d \lambda(\zeta)}{\tau(\zeta, z)^{q} \cdot|\tilde{\Phi}(\zeta, z)|^{n-q}} \preceq\|f\|_{\infty} I_{2}
$$

où

$$
\begin{aligned}
I_{2} & \preceq \int_{0<t_{1}<1} \int_{\left|t_{2}\right|<1} \int_{\left|t^{\prime}\right|<1} \frac{\left|t^{\prime}\right|^{2 n-2 q-3} d t_{1} d t_{2} d\left|t^{\prime}\right|}{\left[t_{1}+\left|t_{2}\right|+t_{1}^{\varepsilon}\left|t^{\prime}\right|^{2}\right]^{n-q}} \\
& \preceq \int_{0<t_{1}<1} \int_{\left|t^{\prime}\right|<1} \frac{d t_{1} d\left|t^{\prime}\right|}{t_{1}^{\alpha+\varepsilon(n-q-1-\alpha)}\left|t^{\prime}\right|^{1-2 \alpha}} \\
& \leq C
\end{aligned}
$$

pour $0<\varepsilon<\frac{1-\alpha}{n-q-1-\alpha}$, avec $\alpha>0$ arbitrairement petit, soit pour tout $\varepsilon, 0<\varepsilon<\frac{1}{n-q-1}$. 


\section{Références}

[C.C] J. Chaumat et A.-M. Chollet, Estimations höldériennes pour les équations de Cauchy-Riemann dans les convexes compacts de $\mathbf{C}^{n}$, Math. Z. 207(4) (1991), 501-534.

[F.R] F. Forelli et W. Rudin, Projections on spaces of holomorphic functions in balls, Indiana Univ. Math. J. 24 (1974/75), 593-602.

[F.S] J. E. Fornæss et N. Sibony, On $L^{p}$ estimates for $\bar{\partial}$, in: "Several complex variables and complex geometry, Part 3 (Santa Cruz, CA, 1989)", Proc. Sympos. Pure Math. 52, Amer. Math. Soc., Providence, RI, 1991, pp. 129-163.

[K] S. G. Krantz, Optimal Lipschitz and $L^{p}$ regularity for the equation $\bar{\partial} u=f$ on stongly pseudo-convex domains, Math. Ann. 219(3) (1976), 233-260.

[P] J. C. Polking, The Cauchy-Riemann equations in convex domains, in: "Several complex variables and complex geometry, Part 3 (Santa Cruz, CA, 1989)", Proc. Sympos. Pure Math. 52, Amer. Math. Soc., Providence, RI, 1991, pp. 309-322.

[R1] R. M. RANGe, On Hölder and BMO estimates for $\bar{\partial}$ on convex domains in $\mathbf{C}^{2}$, J. Geom. Anal. 2(6) (1992), 575-584.

[R2] R. M. RANGE, "Holomorphic functions and integral representations in several complex variables", Graduate Texts in Mathematics 108, Springer-Verlag, New York-Berlin, 1986.

I.U.T. de Nice

Département G.E.A.

41 Boulevard Napoléon III

06041 Nice Cedex

France

Primera versió rebuda el 6 de maig de 1999, darrera versió rebuda el 4 de febrer de 2000. 